\title{
Rotational Isomerism and Nuclear Magnetic Resonance (NMR) Spectra of 1,4-Dibromobutane
}

\author{
DAG F I N N W. AKS NES \\ Chemical Institute, University of Bergen, N-5000 Bergen, Norway
}

\begin{abstract}
Rotamer energy differences and the barrier to rotation of 1,4 dibromobutane have been calculated using a semi-quantitative method.

The 60 and $100 \mathrm{MHz}$ spectra of 1,4-dibromobutane have been recorded and analyzed in terms of chemical shifts and coupling constants. The spectrum of the rapidly reorienting molecule is temperature dependent and constitutes an $\mathrm{A}_{2} \mathrm{BB}^{\prime} \mathrm{B}^{\prime \prime} \mathrm{B}^{\prime \prime \prime} \mathrm{A}_{2}$ ' spin system. Analytical expressions have been obtained for one set of $\mathrm{A}$ and $\mathrm{B}$ 'doublets'. These 'doublets' have the same separation $2\left|J_{\mathrm{AB}}+J_{\mathrm{AB}}\right|$, and are approximately centered about the chemical shift positions. The complete analysis of the spectrum was performed by means of a computer using iterative fitting of experimental and calculated transitions.

Both the theoretical approach and the NMR method predict that the anti form of 1,4-dibromobutane (see Fig. 1) is the more stable. Finally, the calculated spectral parameters have been discussed in relation to the parameters of analogous compounds.
\end{abstract}

\begin{abstract}
$A^{b}$ braham and Parry ${ }^{1}$ have applied a semi-quantitative method for calculating internal rotation barriers and rotamer energy differences in halogenated ethane-like molecules. They obtained barrier heights and energy differences in satisfactory agreement with experimental values. Using their simple model it might be possible to obtain reasonable results for 1,4 -dibromobutane as well.

Many NMR studies have been made on hindered internal rotation about the $\mathrm{C}-\mathrm{C}$ bonds in saturated aliphatic compounds, in particular substituted ethanes., ${ }^{1,2}$ The situation can be complex owing to the increased number of isomers possible. At room temperature, however, the interconversion of the rotational isomers is so rapid that it is not possible to observe the NMR spectra of the separate rotamers. As a consequence, the observed chemical shifts and coupling constants assume average values. The time scale is, of course, set by the NMR experiment. The rotational averaging simplifies the
\end{abstract}


spectrum but reduces its information content. Valuable information can nevertheless be obtained. By lowering the temperature, however, it may be possible to 'freeze out' separate isomers.

The aim of the research reported here was to analyze the complex NMR spectrum of 1,4-dibromobutane. It was furthermore hoped that the NMR method together with the results of the theoretical approach, might give information about the rotational isomers of 1,4-dibromobutane. This investigation is a continuation of the work reported on butane. ${ }^{3}$

\section{CALCULATION OF ROTAMER ENERGIES}

Using substituted cthanes as model ${ }^{2}$ the three staggered rotamers shown in Fig. 1 are the energetically favoured forms of 1,4-dibromobutane. The anti form is unique while the two gauche forms are of equal probability. If the
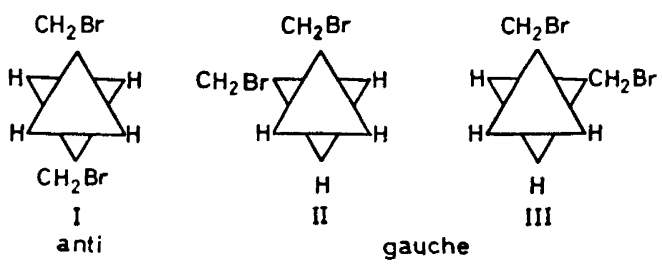

Fig. 1. The rotational isomers of 1,4-dibromobutane.

orientation of the bromine atoms is taken into account, several possible rotamers result. The various rotamers will be specified by italics $a$ (anti) and $g$ (gauche) which refer to the relevant dihedral angles. The rotamer shown below

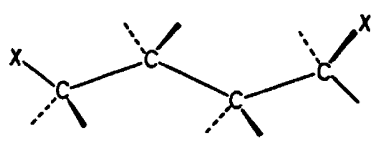

is thus specified as $(a, a, g)$. The first italic refers to the dihedral angle of the $\mathrm{C}_{1}-\mathrm{X}$ bond relative to the central $\mathrm{C}_{2}-\mathrm{C}_{3}$ bond, the second italic refers to the dihedral angle $(\tau)$ of the $\mathrm{C}_{3}-\mathrm{C}_{4}$ bond relative to the $\mathrm{C}_{1}-\mathrm{C}_{2}$ bond, etc.

$\mathrm{By}$ assuming that the various interactions contributing to the rotamer energy $(E)$ are additive, the following equation has been obtained.1

$$
E=\left(V_{0} / 2\right)(1+\cos 3 \tau)+\sum\left(a_{\mathrm{ij}} \exp \left(-b_{\mathrm{ij}} r_{\mathrm{ij}}\right)-c_{\mathrm{ij}} / r_{\mathrm{ij}}{ }^{6}+d_{\mathrm{ij}} / r_{\mathrm{ij}}\right)
$$

The first term in eqn. (1) is the intrinsic torsional barrier. The three terms in the summation of eqn. (1) represent the repulsive and attractive components of the steric interaction, and the electrostatic interaction of the polar substituents, respectively. 
The rotamer energy can be calculated from eqn. (1) once the molecular geometry and the parameters $V_{0}, a, b, c$, and $d$ are known. Using eqn. (1) Abraham and Parry ${ }^{1}$ obtained values of barrier heights and energy differences in fair agreement with the experimental values for a series of substituted ethanes. In the present work, we have used the values quoted by these authors for $V_{0}, a, b, c, d$, bond lengths, and bond angles. ${ }^{1}$ The interatomic distances for a given molecular geometry of 1,4-dibromobutane were calculated using the computer program COORD ${ }^{4}$ which takes as input bond lengths, bond angles, and dihedral angles. A small computer program was written as a subroutine to COORD, in order to calculate the rotamer energy given by eqn. (1). The result of this calculation is listed in Table 1. The calculated energies are relative and have no absolute significance. The calculations show that the $(a, a, a)$ rotamer is the more stable. This seems reasonable on the basis of electron diffraction studies of 1-bromobutane which indicate that the all-anti (zig-zag) conformation is preferred for this compound. ${ }^{5}$

Table 1. Calculated rotamer energies for the energetically favoured forms and one eclipsed conformation of 1,4-dibromobutane (kcal/mol).

\begin{tabular}{|c|c|c|c|c|c|c|}
\hline \multirow{2}{*}{ Rotamer } & \multirow{2}{*}{$\begin{array}{c}\text { Dihedral } \\
\text { angles (deg.) }\end{array}$} & \multicolumn{3}{|c|}{ Calculated contributions } & Total \\
\cline { 3 - 6 } & & Steric & $\mathrm{Br} \cdots \mathrm{Br}$ & $\mathrm{H} \cdots \mathrm{Br}$ & Torsional & \\
\hline & & & & & \\
$(a, a, a)$ & $180,180,180$ & 0.74 & 0.93 & -7.00 & 0 & -5.33 \\
$(a, a, g)$ & $180,180,60$ & 1.19 & 1.15 & -7.34 & 0 & -5.01 \\
$(g, a, g)$ & $60,180,60$ & 1.60 & 1.32 & -7.72 & 0 & -4.80 \\
$\left(g, a, g^{\prime}\right)$ & $60,180,300$ & 1.72 & 1.12 & -7.83 & 0 & -4.98 \\
$(a, g, a)$ & $180,70,180$ & 1.35 & 0.99 & -7.20 & 0.18 & -4.68 \\
$(a, g, g)$ & $180,70,60$ & 1.97 & 1.25 & -7.56 & 0.18 & -4.16 \\
$(g, g, g)$ & $60,70,60$ & 2.75 & 1.20 & -8.11 & 0.18 & -3.98 \\
$(a, e, a)^{a}$ & $180,0,180$ & 3.34 & 1.02 & -7.32 & 2.65 & -0.31 \\
\hline
\end{tabular}

$a$ Eclipsed arrangement $(\tau=0)$.

It is seen from Table 1 that the rotamers having an anti rather than gauche arrangement of the $\mathrm{CH}_{2} \mathrm{Br}$ groups are energetically favoured. This is mainly due to increased interaction between these bulky groups in the gauche forms. The average energy difference between rotamers of the anti and gauche type is about $1.2 \mathrm{kcal} / \mathrm{mol}$. The gauche forms (II and III) have an energy minimum for the dihedral angle $\tau$ equal to $70^{\circ}$ rather than $60^{\circ}$. The energy minimum is, however, broad in this range of dihedral angles. Though the bulky $\mathrm{CH}_{2} \mathrm{Br}$ groups give rise to a large steric contribution, it is clear that polar interactions must be taken into account in order to achieve reasonable results. ${ }^{1}$

It is difficult to calculate true barriers to rotation for 1,4-dibromobutane rigorously since simultaneous rotations will occur about all the $\mathrm{C}-\mathrm{C}$ bonds. It is, however, clear that the rotation is much slower about the central $\mathrm{C}-\mathrm{C}$ bond than about the terminal $\mathrm{C}-\mathrm{C}$ bonds. Defining the barrier height as $E_{0}-E_{60}$ (the suffix represents $\tau$ ) its magnitude can be estimated using eqn. (1). One obtains $E_{0}-E_{60}=4.25 \mathrm{kcal} / \mathrm{mol}$ for a staggered arrangement of the ter- 
minal $\mathrm{CH}_{2} \mathrm{Br}$ groups. This value falls in the range reported for halogen-substituted ethanes and propanes.1,2,6 The actual barrier height is probably smaller because no account has been taken of the fact that the carbon valency angles might relax to relieve steric strain during the formation of the eclipsed arrangement. ${ }^{1,6}$

\section{SPECTRAL ANALYSIS}

The NMR spectrum of 1,4-dibromobutane might be expected to be extremely complex as a consequence of the large number of rotamers possible. However, due to high symmetry of the predominant rotamers and timeaveraging of the NMR spectral parameters, the spectrum is fairly simple. There is no evidence in the NMR spectrum of 1,4-dibromobutane at room temperature that any rotamer is sufficiently long-lived to give its own spectrum. It can therefore be assumed that the reorientations about all the $\mathrm{C}-\mathrm{C}$ bonds are rapid at room temperature as for butane. ${ }^{3}$ The spectrum of the rapidly interconverting molecule is temperature dependent and constitutes an $\mathrm{AA}^{\prime} \mathrm{BB}^{\prime} \mathrm{B}^{\prime \prime} \mathrm{B}^{\prime \prime} \mathrm{A}^{\prime \prime} \mathrm{A}^{\prime \prime}$ spin system.

It is seen from Table 1 that there is a small increase in the rotamer energy when the bromine atom is rotated from an anti to a gauche position at each end of the molecule. It is reasonable to expect a reduction of this energy difference in the liquid state since dipole interactions would tend to stabilize the isomers having the larger dipole moments. This suggests that the NMR spectrum of 1,4-dibromobutane may be analyzed quite accurately as an $\mathrm{A}_{2} \mathrm{BB}^{\prime} \mathrm{B}^{\prime \prime} \mathrm{B}^{\prime \prime \prime} \mathrm{A}_{2}^{\prime}$ spin system. In the following this spin system will be treated theoretically.

It is appropriate at this stage to comment on the spectral parameters involved in the $\mathrm{A}_{2} \mathrm{BB}^{\prime} \mathrm{B}^{\prime \prime} \mathrm{B}^{\prime \prime} \mathrm{A}_{2}^{\prime}$ spin system. $v_{\mathrm{A}}$ and $v_{\mathrm{B}}$ represent the chemical shifts of the magnetically and chemically equivalent $A$ and $B$ nuclei, respectively. There are six coupling constants that affect the spectrum. These are indicated in the diagram:

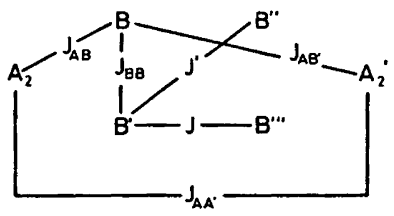

The trans $\left(J_{t}\right)$ and gauche $\left(J_{g}\right)$ coupling constants of the methylene hydrogens in the fixed rotamers will assume average values $J^{\prime}$ and $J$, respectively. The analysis gives the average value of $J^{\prime}$ and $J$ which can easily be expressed in terms of $J_{t}$ and $J_{g}$ (see Fig. 1)

$$
\begin{aligned}
& J^{\prime}=(1-p) J_{g}+p J_{t} \\
& J=\frac{1}{2}(1+p) J_{g}+\frac{1}{2}(1-p) J_{t}
\end{aligned}
$$

where $p$ is the relative weight of the anti form (I). Eqn. (2) follows from the assumption that $J_{t}$ and $J_{g}$ are invariant for a given compound. This is, of

Acta Chem. Scand. 26 (1972) No. 1 
course, an approximation,,$^{2,7}$ particularly since the dihedral angles between the $\mathrm{C}-\mathrm{H}$ bonds in the ethanic fragment might differ appreciably from $60^{\circ}$ and $180^{\circ}$ in the gauche form. Putting $\Delta E=E_{g}-E_{a}$ and assuming that the rotamers have the same partition function, it is found that ${ }^{2}$

$$
p=\frac{1}{1+2 \exp (-\Lambda E / R T)}
$$

in which it is implicit that $E_{g}>E_{a}$. The chemical shifts would have to be averaged in a similar manner if they were to be used in the calculation of rotamer populations.

The $\mathrm{A}_{2} \mathrm{BB}^{\prime} \mathrm{B}^{\prime \prime} \mathrm{B}^{\prime \prime} \mathrm{A}_{2}^{\prime}$ spin system can be treated theoretically by the composite particle technique. ${ }^{8}$ The following spin states are possible:

\section{TDDDDT}

\section{TDDDDS SDDDDT \\ SDDDDS}

In this notation the symbols $\mathrm{T}, \mathrm{D}$, and $\mathrm{S}$ represent the composite particle states triplet, doublet, and singlet having total spin quantum numbers $F$ equal to $1, \frac{1}{2}$, and 0 , respectively. It is convenient to define a magnetic quantum number $m_{\mathrm{r}}=\sum_{\mathrm{i}} m_{\mathrm{i}}$ where the summation is over the six composite particle
states.

The total relative intensities attributable to the four spin states TDDDDT, TDDDDS, SDDDDT, and SDDDDS are 672, 160, 160, and 32, respectively. The latter spin state only gives rise to extra intensity at $v_{\mathrm{B}}$. The pair of states TDDDDS and SDDDDT are degenerate and cannot mix. Since it can be assumed that the time-averaged five-bond coupling $J_{\mathrm{AA}^{\prime}}=0$, all the spectral parameters may be obtained from the degenerate sub-spectrum.

The TDDDDT sub-spectrum is, however, easier to treat theoretically since the secular matrices can be reduced by considering additional symmetry not included in the composite particle notation, viz. ${ }^{8}$

$$
\mathrm{TDDDDT}=\mathrm{TDDDDT}(\mathrm{s})+\mathrm{TDDDDT}(\mathrm{a})
$$

where (s) and (a) indicate symmetrical and antisymmetrical wave functions. The sub-Hamiltonian of the TDDDDT sub-spectrum is thus factorized into energy level diagrams with groupings (1:3:9:15:18:15:9:3:1) and (3:8:15:18:15:8:3) for the symmetrical and antisymmetrical wave functions, respectively. A partial

Table 2. Partial energy level diagram for the TDDDDT(s) sub-system of the $\mathrm{A}_{2} \mathrm{BB}^{\prime} \mathrm{B}^{\prime \prime} \mathrm{B}^{\prime \prime \prime} \mathrm{A}_{2}^{\prime}$ spin system.

$$
\begin{array}{llll}
m_{\mathrm{T}} & & \\
4 & & \underline{E_{4}} \\
3 & \underline{E_{31}} & \underline{E_{32}} & \underline{E_{33}} \\
. & & & \\
\cdot & & & \\
-3 & \underline{E_{-31}} & \frac{E_{-32}}{-4} & \underline{E_{-33}} \\
-E_{-4} &
\end{array}
$$

Acta Chem. Scand. 26 (1972) No. 1 
energy level diagram for the TDDDDT(s) sub-system is indicated schematically in Table 2. The only off-diagonal elements are $(1 / \sqrt{2})\left(J_{\mathrm{AB}}+J_{\mathrm{AB}^{\prime}},\right)$ and $\frac{1}{2}\left(J_{\mathrm{BB}}+J^{\prime}\right)$.

Expressions in analytical form can now be obtained for the energy levels corresponding to $m_{\mathrm{T}}= \pm 3$ since this only involves solving secular equations of second order. Then the frequencies of the six transitions $m_{\mathrm{r}}=3 \rightarrow 4$ and $m_{\mathrm{r}}=-4 \rightarrow-3$ also give closed expressions. Four of these transitions have high intensity while the remaining two $B$ transitions are forbidden. This can be seen by taking account of the full $C_{2 v}$ symmetry of this system.

After some tedious algebra the following expressions are obtained for the energy levels (omitting terms in $J_{\mathrm{AA}^{\prime}}$ ).

$$
\begin{aligned}
& E_{4}=2 v_{\mathrm{A}}+2 v_{\mathrm{B}}+2 \mathrm{~A}+\mathrm{B} \\
& E_{31}=2 v_{\mathrm{A}}+v_{\mathrm{B}}+\mathrm{A}+\frac{1}{2}\left(J-J^{\prime}-J_{\mathrm{BB}}\right) \\
& E_{32}=\frac{1}{2}\left[3\left(v_{\mathrm{A}}+v_{\mathrm{B}}\right)+2 \mathrm{~A}+2 \mathrm{~B}+\mathrm{C}\right] \\
& E_{33}=\frac{1}{2}\left[3\left(v_{\mathrm{A}}+v_{\mathrm{B}}\right)+2 \mathrm{~A}+2 \mathrm{~B}-\mathrm{C}\right]
\end{aligned}
$$

where $\mathrm{A}=J_{\mathrm{AB}}+J_{\mathrm{AB}}{ }^{\prime} ; \mathrm{B}=\frac{1}{2}\left(J+J^{\prime}+J_{\mathrm{BB}}\right) ; \mathrm{C}=\left[\left(v_{\mathrm{A}}-v_{\mathrm{B}}\right)^{2}+4 \mathrm{~A}^{2}\right]^{\frac{1}{2}}$

Corresponding expressions are obtained for $E_{-4}$ and $E_{-3}$ by replacing $\nu_{\mathrm{A}}$ and $v_{\mathrm{B}}$ by $-v_{\mathrm{A}}$ and $-v_{\mathrm{B}}$, respectively. The two strong $\mathrm{A}$ transitions have frequencies

$$
\begin{aligned}
& E_{4}-E_{33}=v_{\mathrm{A}}+\mathrm{A}+\frac{1}{2}\left(\mathrm{C}-\left|\nu_{\mathrm{A}}-v_{\mathrm{B}}\right|\right) \\
& E_{-32}-E_{-4}=v_{\mathrm{A}}-\mathrm{A}+\frac{1}{2}\left(\mathrm{C}-\left|v_{\mathrm{A}}-v_{\mathrm{B}}\right|\right)
\end{aligned}
$$

with a separation equal to $2|\mathbf{A}|$. The two strong $B$ transitions occur at the frequencies

$$
\begin{aligned}
& E_{4}-E_{32}=v_{\mathrm{B}}+\mathrm{A}-\frac{1}{2}\left(\mathrm{C}-\left|v_{\mathrm{A}}-v_{\mathrm{B}}\right|\right) \\
& E_{-33}-E_{-4}=v_{\mathrm{B}}-\mathrm{A}-\frac{1}{2}\left(C-\left|v_{\mathrm{A}}-v_{\mathrm{B}}\right|\right)
\end{aligned}
$$

These two lines are also separated by $2|\mathbf{A}|$. Since $\left|v_{\mathrm{A}}-v_{\mathrm{B}}\right| \gg|\mathrm{A}|$ in the present case, it follows that $\mathrm{C} \simeq\left|v_{\mathrm{A}}-v_{\mathrm{B}}\right|$. It is then seen from eqns. (5) and (6) that the A and B 'doublets' should be observed near $v_{\mathrm{A}} \pm \mathrm{A}$ and $v_{\mathrm{B}} \pm \mathrm{A}$, respectively (see Figs. 4 and 5).

The remaining transitions cannot be obtained in analytical form since they are connected to energy levels having $m_{\mathrm{r}}= \pm 2, \pm 1,0$, and would require solving secular matrices of at least third order even if account is taken of the $C_{2 v}$ symmetry.

It is easy to pick out the A and B 'doublets' from the experimental spectra since the two 'doublets' have the same separation $2|\mathrm{~A}|$ and are approximately centered about $\nu_{\mathrm{A}}$ and $\nu_{\mathrm{B}}$ (see Figs. 2-5). The 60 and $100 \mathrm{MHz}$ spectra yield the same value of $2|\mathrm{~A}|$ to the second decimal place. From the $\mathrm{A}$ and $\mathrm{B}$ spectra it follows that $2|\mathrm{~A}|=12.69 \mathrm{~Hz}$ and $12.67 \mathrm{~Hz}$, respectively.

A complete analysis of the NMR spectrum using iterative fitting of experimental and calculated transitions has been performed by means of the computer program LACX $^{\mathbf{9}, 10}$. Since this program takes account of chemical equivalence, the NMR spectrum can be analyzed as a four-spin system. The computation is therefore very rapid compared to the LAOCN3 ${ }^{11}$ program which takes no account of symmetry. 'Stick'-plots of spectra calculated from trial parameters were obtained using the computer program KOMBIP.11

Act Chem. Scand. 26 (1972) No. 1 
The spectrum was first analyzed as an $\mathrm{A}_{2} \mathrm{BB}^{\prime} \mathrm{B}^{\prime \prime} \mathrm{B}^{\prime \prime} \mathrm{A}_{2}{ }^{\prime}$ spin system. Trial values of the coupling constants were estimated from the known value of $\left|J_{\mathrm{AB}}+J_{\mathrm{AB}}\right|$ and from similar parameters of analogous compounds. A total of fifty-six well-resolved lines were measured in the experimental spectra. However, due to the large number of degenerate transitions, about two hundred transitions were matched. The root-mean-square deviations were 0.09 and $0.06 \mathrm{~Hz}$ for the 60 and $100 \mathrm{MHz}$ spectra, respectively. The calculated standard deviations were about $0.01 \mathrm{~Hz}$. The error is, however, larger in the geminal coupling constant, $J_{\mathrm{BB}}$, because the spectra were insensitive to variation of this parameter. The calculated spectral parameters are listed in Table 3.

The experimental and computed $60 \mathrm{MHz}$ proton spectra of 1,4-dibromobutane are shown in Figs. 2 and 3 . 'Stick-plots of the total $\mathrm{A}_{2} \mathrm{BB}^{\prime} \mathrm{B}^{\prime \prime} \mathrm{B}^{\prime \prime \prime} \mathrm{A}_{2}$ ' spectrum and its composite TDDDDT sub-spectrum are displayed in Figs. 4 and 5 . The intensity scale is the same for these plots.

The spectrum was then analyzed as an $\mathrm{AA}^{\prime} \mathrm{BB}^{\prime} \mathrm{B}^{\prime \prime} \mathrm{B}^{\prime \prime} \mathrm{A}^{\prime \prime} \mathrm{A}^{\prime \prime}$ spin system using the same computer program. $J_{\mathrm{AB}}$ and $J_{\mathrm{AB}^{\prime}}$ were then both replaced by averaged trans and gauche coupling constants similar to those in eqn. (2). The corresponding trans and gauche coupling constants were, however, found

Table 3. NMR spectral parameters at 60 and $100 \mathrm{MHz}$ of 1,4 -dibromobutane in respectively $25 \% \mathrm{v} / \mathrm{v}$ solution (first entry) and as neat liquid (second entry). ${ }^{a}$

\begin{tabular}{|r|r|c|c|c|c|c|c|c|}
\hline$v_{0}^{v_{0}}$ & $v_{\mathrm{A}}-v_{\mathrm{B}}$ & $\begin{array}{c}\delta_{\mathrm{AB}} \\
(\mathrm{ppm})\end{array}$ & $J_{\mathrm{AB}}$ & $J_{\mathrm{AB}^{\prime}}$ & $J_{\mathrm{AA}^{\prime}}$ & $J_{\mathrm{BB}}{ }^{b}$ & $J$ & $J^{\prime}$ \\
\hline 60 & 85.99 & 1.433 & 6.64 & -0.22 & 0 & -11.2 & 5.44 & 9.47 \\
100 & 144.52 & 1.445 & 6.61 & -0.25 & 0 & -11.2 & 5.44 & 9.46 \\
\hline
\end{tabular}

a Spectral parameters in $\mathrm{Hz}$ if not otherwise stated.

${ }^{b}$ Not varied in the final iteration.

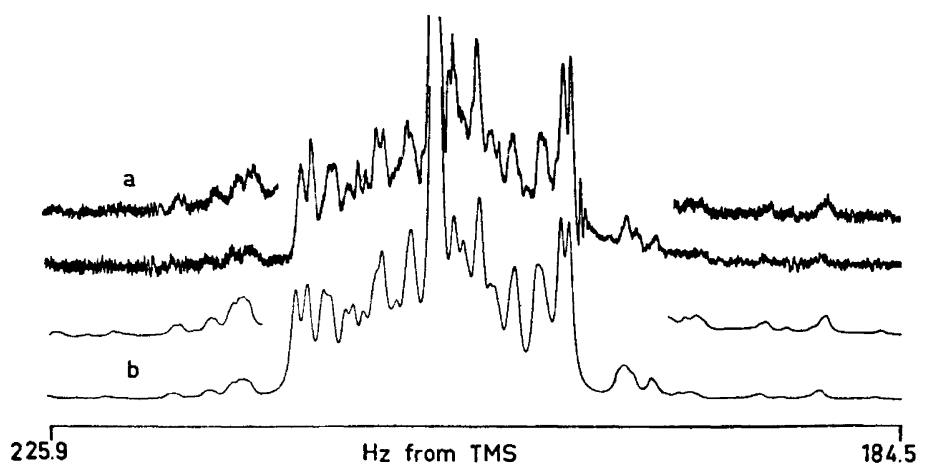

Fig. 2. The $60 \mathrm{MHz}$ bromomethyl proton spectrum (A part of the $\mathrm{A}_{2} \mathrm{BB}^{\prime} \mathrm{B}^{\prime \prime} \mathrm{B}^{\prime \prime} \mathrm{A}_{2}^{\prime}$ spin system) of 1,4-dibromobutane; a, observed; b, computed (half-width $0.4 \mathrm{~Hz}$ ). 


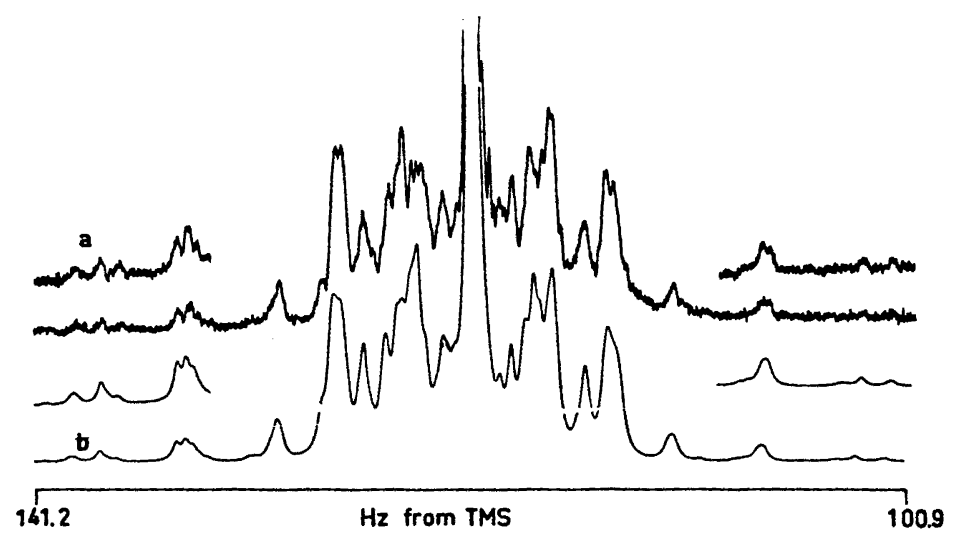

Fig. 3. The $60 \mathrm{MHz}$ methylene proton spectrum (B part of the $\mathbf{A}_{2} \mathbf{B B}^{\prime} \mathbf{B}^{\prime \prime} \mathbf{B}^{\prime \prime} \mathbf{A}_{2}^{\prime}$ spin system) of 1,4-dibromobutane; a, observed; b, computed (half-width $0.4 \mathrm{~Hz}$ ).

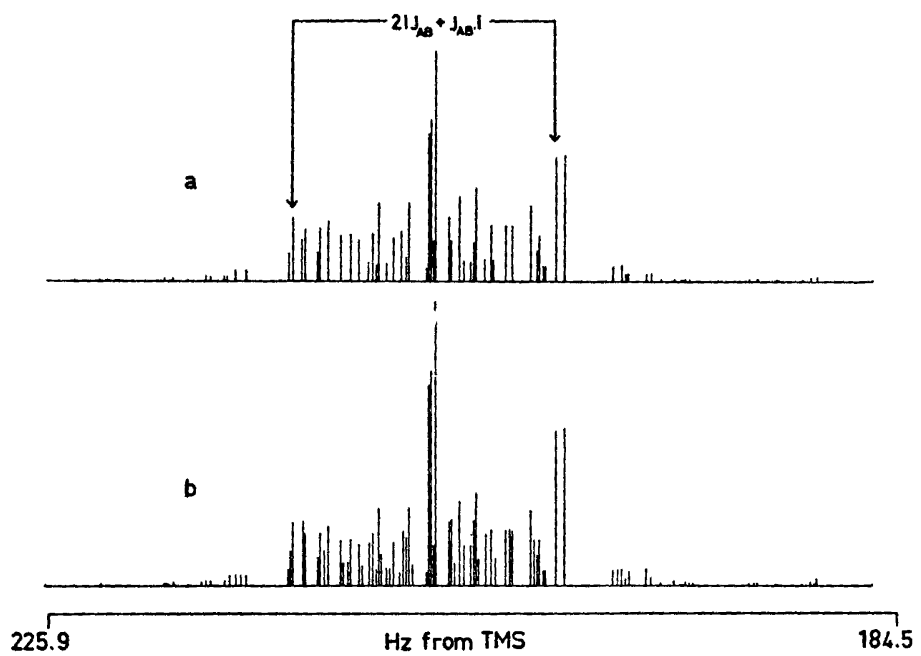

Fig. 4. Computed $60 \mathrm{MHz}$ bromomethyl proton spectrum (A part of the $\mathrm{A}_{2} \mathrm{BB}^{\prime} \mathrm{B}^{\prime \prime} \mathrm{B}^{\prime \prime \prime} \mathrm{A}_{2}^{\prime}$ spin system) of 1,4-dibromobutane; $a$, composite TDDDDT sub-spectrum; $b$, total spectrum. The intensity scale is the same for these plots.

to differ by less than the sum of the standard deviations for the individual coupling constants. Furthermore, the remaining parameters retained the same values. This then supports the assumption concerning the type of spin system.

Acta Chem. Scand. 26 (1972) No. 1 


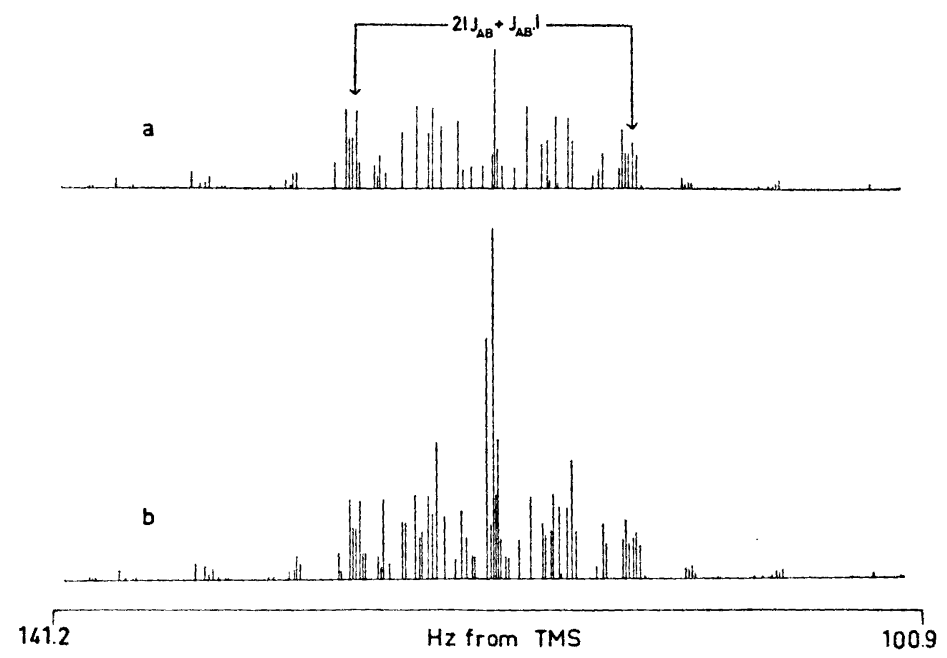

Fig. 5. Computed $60 \mathrm{MHz}$ methylene proton spectrum (B part of the $A_{2} B^{\prime} B^{\prime \prime} B^{\prime \prime \prime} A_{2}^{\prime}$ spin system) of 1,4-dibromobutane; $a$, composite TDDDDT sub-spectrum; $b$, total spectrum. The intensity scale is the same as in Fig. 4.

\section{DISCUSSION}

The agreement between the experimental and computed spectra is very good (see Figs. 2 and 3). The excellent fit between observed and calculated transitions at the wings of the spectra should, in particular, be noticed.

The agreement between the spectral parameters obtained at 60 and 100 $\mathrm{MHz}$ is very good. The small deviations can be attributed to experimental error and medium effects. The molecular association is seen to have a larger effect on the chemical shift than on the coupling constants, as expected. The magnitude of the observed chemical shift is about 1 ppm larger than for butane ${ }^{3}$ and propane, ${ }^{13-15}$ and reversed in sign. This is as expected for saturated hydrocarbons possessing terminal electronegative substituents.

The energy difference, $\Delta E$, between the anti and gauche rotamers can be calculated using eqns. (2) and (3) provided that either $J_{t}$ or $J_{g}$ is known. The experimental result $J_{t}>J_{g}$ is now well-established in hydrocarbon groupings. ${ }^{2}$ The substituent effect of the bromine atoms on $J_{t}$ and $J_{g}$ is probably small because these atoms are two bonds away from the ethanic fragment. It can therefore be assumed that these coupling constants have about the same values as in butane. ${ }^{3}$ Using the reasonable value $J_{t}=11 \mathrm{~Hz}$ it follows from eqns. (2) and (3) that $\Delta E=1.1 \mathrm{kcal} / \mathrm{mol}$ and $J_{g}=4.7 \mathrm{~Hz}$. This gives $J_{t}+2 J_{g}=$ $20.4 \mathrm{~Hz}$ which is fairly close to the values reported for similar compounds. ${ }^{2,3}$

The magnitude of $\Delta E$ obtained by the NMR method is close to the value predicted theoretically. The latter value, however, is in the gas phase whilst the former value is obtained in the liquid phase where dipole interactions would tend to stabilize the rotamers having the larger dipole moments. The 
two approaches, nevertheless, predict that the anti form (I) predominates. Steric and polar interactions make the major contribution to the energy difference. It is clear that the electrostatic interactions must be taken into account in order to achieve reasonable results. ${ }^{1}$

By observing the effect of temperature changes on the spectral parameters, distinguished values of $J_{t}$ and $J_{g}$ for the anti and gauche form could be obtained. ${ }^{7}$ This refined analysis would also yield a more accurate value of $\Delta \mathrm{E}$.

The vicinal coupling constant, $J_{\mathrm{AB}}$, is the average of the coupling constants in the various rotamers. The magnitude is close to the corresponding coupling constants in 1,2-dibromopropane ${ }^{16}(6.49 \mathrm{~Hz})$ and meso and $\mathrm{DL}_{4}-2,3$-dibromobutane $^{17}$ (6.54 and $6.74 \mathrm{~Hz}$, respectively) in dilute solutions. The vicinal coupling constant is, however, considerably larger in propane ${ }^{13-15}(7.45 \mathrm{~Hz})$ and butane ${ }^{3}(7.40 \mathrm{~Hz})$, as expected. The vicinal couplings are affected by substituents on the $\mathrm{CH}-\mathrm{CH}$ fragment in a very similar manner to the olefinic couplings.

The geminal coupling constant does not merit a detailed discussion owing to large experimental error. The sign was, however, observed to be negative. This is typical of saturated aliphatic systems.

The long-range coupling constant, $J_{\mathrm{AB}^{\prime}}$, is relatively small due to rotational averaging. Motionally averaged four-bond couplings of similar magnitude have been measured in saturated aliphatic and cyclic compounds. ${ }^{18}$ Replacement of hydrogen by an inductive substituent on the terminal carbon atom of a propanic fragment is expected to cause a decrease in the four-bond coupling. ${ }^{18}$ Due to the conformational dependence of $J_{\mathrm{AB}^{\prime}}$ it is, however, impossible to ascribe the small differences observed in $J_{\mathrm{AB}^{\prime}}$ for the analogous compounds butane, ${ }^{3}$ 1,4-dibromobutane, propane, ${ }^{13-15}$ and 1,2-dibromopropane ${ }^{16}$ to substituent effects. The four-bond coupling constants can be of either sign depending on the relative orientation of the coupled protons, and timeaveraging. 18

\section{EXPERIMENTAL}

1,4-Dibromobutane was obtained from Fluka AG. The liquid was redistilled under vacuum for further purification. The 60 and $100 \mathrm{MHz}$ NMR spectra were examined in methylenechloride solution $(25 \% \mathrm{v} / \mathrm{v})$ and as neat liquid, respectively. The resolution was improved on dilution in $\mathrm{CH}_{2} \mathrm{Cl}_{2}$. Tetramethylsilane was used as internal reference and lock signal source. The samples were thoroughly degassed and sealed under vacuum.

The 60 and $100 \mathrm{MHz}$ spectra were run at ambient temperatures $\left(27\right.$ and $30^{\circ} \mathrm{C}$, respectively) on JEOL-C-60H and VARIAN HA-100 spectrometers. No impurities were detectable in the measured range. The spectra used in the calculation were recorded at 54 and $50 \mathrm{~Hz}$ sweep widths and calibrated every $5 \mathrm{~Hz}$, using a frequency-counter accurate to $\pm 0.1 \mathrm{~Hz}$. Line positions were obtained by averaging the results of six scans.

Computations were performed on the IBM $360 / 50 \mathrm{H}$ computer at the University of Bergen. Graphical plots were obtained using a Calcomp Plotter.

Acknowledgement. The author is indebted to Professor J. Dale at the Chemical Institute, University of Oslo, Norway, for the opportunity to use the VARIAN HA-100 spectrometer at his laboratory.

\section{REFERENCES}

1. Abraham, R. J. and Parry, K. J. Chem. Soc. B 1970 539, and references therein.

2. Emsley, J. W., Feeney, J. and Sutcliffe, L. H. High Resolution Nuclear Magnetic

Acta Chem. Scand. 26 (1972) No. 1 
Resonance Spectroscopy, Vol. 1, p. 559, Pergamon, New York 1966, and references therein.

3. Aksnes, D. W. and Albriktsen, P. Acta Chem. Scand. 24 (1970) 3764.

4. COORD, Program No. 136, Quantum Chemistry Program Exchange, Indiana University, Chemistry Department, Indiane, USA.

5. Momany, F. A., Bonham, R. A. and MeCoy, W. H. J. Am. Chem. Soc. 85 (1963) 3077.

6. Newmark, R. A. and Sederholm, C. H. J. Chem. Phys. 43 (1965) 602.

7. Harris, R. K. and Sheppard, N. Trans. Faraday Soc. 59 (1963) 606.

8. Diehl, P., Harris, R. K. and Jones, R. G. Progr. NMR Spectr. 3 (1967) 1.

9. Haigh, C. W. LACX, Department of Chemistry, University College, Swansea, England.

10. Haigh, C. W. J. Chem. Soc. A 19701682.

11. Bothner-By, A. A. and Castellano, S. LAOCN3, Mellon Institute, Pittsburgh, Pennsylvania, USA 1966.

12. Aksnes, D. W. KOMBIP, Chemical Institute, University of Bergen, Bergen, Norway. (The program is available on request.)

13. Onsager, L., Saunders, M. and Dubb, H. E. J. Chem. Phys. 32 (1960) 67.

14. Sheppard, N. and Turner, J. J. Mol. Phys. 3 (1960) 168.

15. Corio, P. L. and Hirst, R. C. J. Chem. Educ. 46 (1969) 345.

16. Finegold, H. Proc. Chem. Soc. 1962213.

17. Anet, F. A. L. J. Am. Chem. Soc. 84 (196٪) 747.

18. Barfield, M. and Chakrabarti, B. Chem. Rev. 69 (1969) 757, and references therein.

Received March 24, 1971. 